\title{
Quantum Monte Carlo Study of a Disordered 2-D Josephson Junction Array
}

\author{
W. A. Al-Said: and D. Stroudt \\ Department of Physics, The Ohio State University, Columbus, Ohio 43210
}

(Dated: November 13, 2018)

\begin{abstract}
We have studied the superconducting-insulating phase transition in a disordered two-dimensional Josephson junction array, using quantum Monte Carlo techniques. We consider disorder in both the capacitive energies and in the values of the offset charges. The calculated phase diagram shows that the lobe structure of the phase diagram disappears for sufficiently strong disorder in the offset charge. Our results agree quite well with previous calculations carried out using a mean-field approximation.
\end{abstract}

PACS numbers: 74.25.Dw, 05.30.Jp, 85.25.Cp 


\section{INTRODUCTION}

A Josephson junction array (JJA) consists of a collection of superconducting islands connected by Josephson coupling. The coupling can arise from tunnel junctions through an insulating layer, or from the proximity effect. The arrays themselves can be produced experimentally in a wide range of geometries, and with a great variety of individual junction parameters. They can serve as valuable model systems for studying quantum phase transitions ${ }^{1.2}$ under conditions such that the experimental parameters can be readily tuned ${ }^{3}$. Recently, it has also been proposed that small groups of Josephson junctions may serve as quantum bits (qubits) in quantum information technology. In this case, the quantum logic operations are performed by manipulating experimental parameters such as gate voltages or magnetic fields ${ }^{4}$.

If the superconducting islands in a Josephson array are sufficiently large, the array is believed to become superconducting in two stages. First, at a temperature near the bulk transition temperature, each island becomes superconducting. Secondly, at a lower temperature, and provided that the array is at least two-dimensional, thermal fluctuations become sufficiently weak that a global phase coherence can be established throughout the array ${ }^{2}$.

If, however, the islands in the array are small, such phase coherence may not be established even at temperature $T=0$. The reason is that there is a second energy which becomes important in small grains, namely the charging energy of the grains $s^{\frac{5}{}}$. The crucial physics is then determined by the competition between this charging energy and the Josephson coupling energy. If the charging energy is sufficiently large, then it becomes prohibitively expensive energetically to transfer Cooper pairs from grain to grain, and the array becomes an insulator, even though each grain is in its superconducting state. If, on the other hand, the Josephson dominates, the array becomes phase coherent at $T=0$. In this case, Cooper pairs can tunnel between neighboring grains and the array as a whole will be in the superconducting phase. Thus, by tuning the ratio of the charging and Josephson energies, one can cause the array to undergo a quantum phase transition between a superconducting and an insulating state ${ }^{1.3}$.

In the limit of very large number of Cooper pairs per grain, the Josephson junction array with diagonal charging energy is equivalent to the Bose Hubbard model (BHM), which describes soft core bosons hopping on a lattice with on-site Coulomb interactions. The 
BHM has previously been extensively studied by Fisher et al $\stackrel{8}{\underline{8}}$, who constructed its $T=0$ phase diagram. In the absence of disorder, the phase diagram shows two phases: a Mott insulating phase and a superfluid phase. In the presence of disorder, an additional phase,

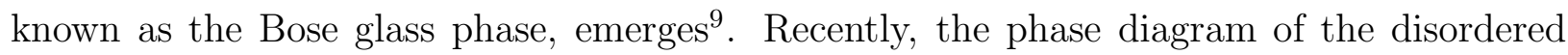
BHM in 2-dimensions (2D) has been studied by Lee et al using QMCํㅗ.

The aim of this paper is to construct the phase diagram for the superconducting-insulating (SI) transition in a particular model for a JJA, including the effects of disorder. Such disorder is clearly unavoidable in most practical systems. The model Hamiltonian we study has previously been investigated by several authors in ordered and disordered arrays, using both mean-field theory (MFT) and quantum Monte Carlo (QMC) techniques. A central goal of our work is to compare the MFT of Refs $\frac{6.7}{6}$ and QMC results in a disordered array in order to check the accuracy of the MFT. We shall show that the MFT is generally satisfactory, even for disordered systems.

The remainder of this paper is organized as follows. In the next section, we present the model Hamiltonian and describe the QMC algorithm we use. Our numerical results are presented in Section III, followed by a brief concluding discussion in Section IV.

\section{MODEL HAMILTONIAN AND QMC ALGORITHM}

Our model of a JJA Hamiltonian involves two types of degrees of freedom: the number of excess Cooper pairs $\hat{n}_{i}$ on the $i$ th grain, and the phase $\hat{\phi}_{i}$ of the superconducting order parameter on the $i$ th grain. $\hat{n}_{i}$ and $\hat{\phi}_{i}$ are taken to be quantum-mechanically conjugate variables with commutation relations $\left[\hat{n}_{i}, \hat{\phi}_{j}\right]=-i \delta_{i, j}$. We consider the following model Hamiltonian on a square lattice in 2D:

$$
\mathcal{H}=\frac{1}{2} \sum_{i} U_{i i}\left(\hat{n}_{i}-\bar{n}_{i}\right)^{2}-E_{J} \sum_{\langle i, j\rangle} \cos \left(\hat{\phi}_{i}-\hat{\phi}_{j}\right) .
$$

Here $E_{J}$ is the Josephson coupling strength between nearest neighbors denoted by $\langle i, j\rangle$ (assumed to be the same for all nearest neighbor pairs), and $U_{i i}$ is the charging energy of the $i$ th grain. We expect that $U_{i i}=q^{2} / C_{i i}$, where $C_{i i}$ represents the capacitance of the $i$ th grain with respect to ground, and $q=2 e$ is the charge of a Cooper pair. In a more general model, the charging part of the energy would be written $\frac{1}{2} U_{i j}\left(\hat{n}_{i}-\bar{n}_{i}\right)\left(\hat{n}_{j}-\bar{n}_{j}\right)$, where $U_{i j}$ is an element of the charging energy matrix. In earlier calculations using MFT, nearest-neighbor 
and next-nearest-neighbor terms have been included, but these are numerically more difficult to include in QMC than the diagonal terms. Finally, the quantity $\bar{n}_{i}$ represents the "offset charge." $\bar{n}_{i}$ is related to the voltage between the $i$ th grain and a common ground plane. In an ordered array, $\bar{n}_{i}$ should be independent of $i$. We omit any dissipative terms arising from Ohmic shunts in the junctions, and we assume that amplitude fluctuations of the gap on the individual grains can be neglected; this neglect of amplitude fluctuations should be reasonable if $T \ll T_{c 0}$, the single-grain transition temperature. Thus tunneling of charge between grains involves only Cooper pairs, not single electrons.

In our calculations, we include disorder in two terms in the Hamiltonian: the diagonal charging element $U_{i i}$ and the offset charge $\bar{n}_{i}$. Disorder in $U_{i i}$ may arise from randomness in the size of the individual grains, while disorder in $\bar{n}_{i}$ could arise from random offset charges near the superconducting grains. Such disorder is unavoidable in practical realizations of Josephson arrays.

It is worthwhile to discuss the effects of the offset charges $\bar{n}_{i}$ qualitatively. Such charges can arise in two ways: (i) from a voltage applied between the array and the substrate, and (ii) from random charges in the substrate. In the latter case, $\bar{n}_{i}$ is a random variable. In arrays with only a few grains, $\bar{n}_{i}$ can, in principle, be tuned individually to desired values. However, such tuning, although possibly still achievable in principle, may be difficult to attain in practice in arrays with many junctions.

In ordered arrays, the most important effect of the offset charge is to reduce the region of the phase diagram where the Mott insulating phase is stable. The reason is that, if $\bar{n}_{i} \neq 0$, charge fluctuations become energetically less expensive; it is therefore correspondingly easier to establish phase coherence and to destroy the Mott insulator.

For the disordered array, we determine the phase diagram of the Hamiltonian (II) using QMC techniques $11,12.13$, and compare the results with MFT $\underline{\underline{7}}$. Following the method of Ref. $\underline{\underline{11}}$, we first map the 2D Hamiltonian Hamiltonian $\mathcal{H}$ [Eq. (1)], onto a classical 3D Hamiltonian. This is accomplished by the standard procedure of transforming the partition function $Z=$ $\operatorname{Tr} e^{-\beta \mathcal{H}}$, where $\beta=1 / k_{B} T$, into an Euclidean path integral along the imaginary time axis $\tau$ from 0 to $\hbar \beta$. To do the transformation, one breaks up the time integral into $L_{\tau}$ small time steps, each of length $\epsilon=\hbar \beta / L_{\tau}$, and uses the identity $e^{-\beta \mathcal{H}}=e^{-\epsilon \mathcal{H}} \ldots e^{-\epsilon \mathcal{H}}$. Next, a complete set of eigenstates (for example, the eigenstates of the Cooper pair number operator for the $i$ th grain) are inserted at each imaginary time step. The next step is to rewrite the 
Josephson term using the Villain approximation $\frac{14}{\underline{14}}$ as $e^{-x \cos \phi} \approx \sum_{m=-\infty}^{\infty} e^{-x f(x)(\phi-2 \pi m)^{2} / 2}$, where $f(x)=\left\{2 x \ln \left[I_{0}(x) / I_{1}(x)\right]\right\}^{-1}$, and $I_{n}(x)$ is the modified Bessel function of order $n$. For large values of $x, f(x) \approx 1$.

Finally, the partition function $Z$ can be expressed as $Z=\operatorname{Tr} e^{-S}$, where the action $S$ is defined as

$$
\begin{aligned}
S=\frac{1}{2} \epsilon \sum_{i, j, \tau} & U_{i, j}\left(J_{i, \tau}^{(\tau)}-\bar{n}_{i, \tau}\right)\left(J_{j, \tau}^{(\tau)}-\bar{n}_{j, \tau}\right) \\
& +\frac{1}{2 E_{J} \epsilon f\left(E_{J} \epsilon\right)} \sum_{i, \tau, \alpha=x, y}\left|J_{i, \tau}^{(\alpha)}\right|^{2} .
\end{aligned}
$$

In Eq. (2), the new degrees of freedom are integer-valued current loops $J_{i, \tau}^{(\alpha)}(\alpha=x, y)$, which live on the nodes of the 3-dimensional $(\mathrm{xy} \tau)$ lattice, and satisfy the continuity equation $\sum_{\alpha=x, y}, \partial_{\alpha} J_{i, \tau}^{(\alpha)}+\partial_{\tau} J_{i, \tau}^{(\tau)}=0$ at every lattice point. The time components of the current operators, $J_{i, \tau}^{(\tau)}$, represent the Cooper pair number operators $n_{i, \tau}$ along their world lines. Note that $S$ is a classical action in $(d+1)$ dimensions while the original $\mathcal{H}$ is a quantum Hamiltonian in $d$ dimensions.

To evaluate $S$, we use the standard Metropolis algorithm to generate configurations of the currents $\mathbf{J}_{i, \tau}$ at inverse temperature $\beta$. It is convenient to work in the grand canonical ensemble. The system size is assumed to be a parallelepiped with dimensions $\left(L, L, L_{\tau}\right)$ along the space and imaginary time axes respectively, with periodic boundary conditions in all three directions.

We locate the superconducting-insulating (SI) phase transition by examining the superfluid density $\rho$, which is proportional to the stiffness of the system against a twist in the phase. As has been shown previously $\underline{\underline{11}}, \rho$ can be expressed in terms of the current variables $\mathbf{J}_{i, \tau}$ :

$$
\rho=\frac{1}{L^{2} L_{\tau}}\left\langle\left\langle w^{(x)}\right\rangle\right\rangle
$$

Here $w^{(x)}=\left|\sum_{i, \tau} J_{i, \tau}^{(x)}\right|^{2}$ is the so-called winding number in the x-direction, and $\langle\langle\cdots\rangle\rangle$ denotes both a grand canonical and a disorder average. If $\rho$ is non-zero in the thermodynamic limit of a large system, then there is long-range phase coherence and the system will be in the superconducting phase. 


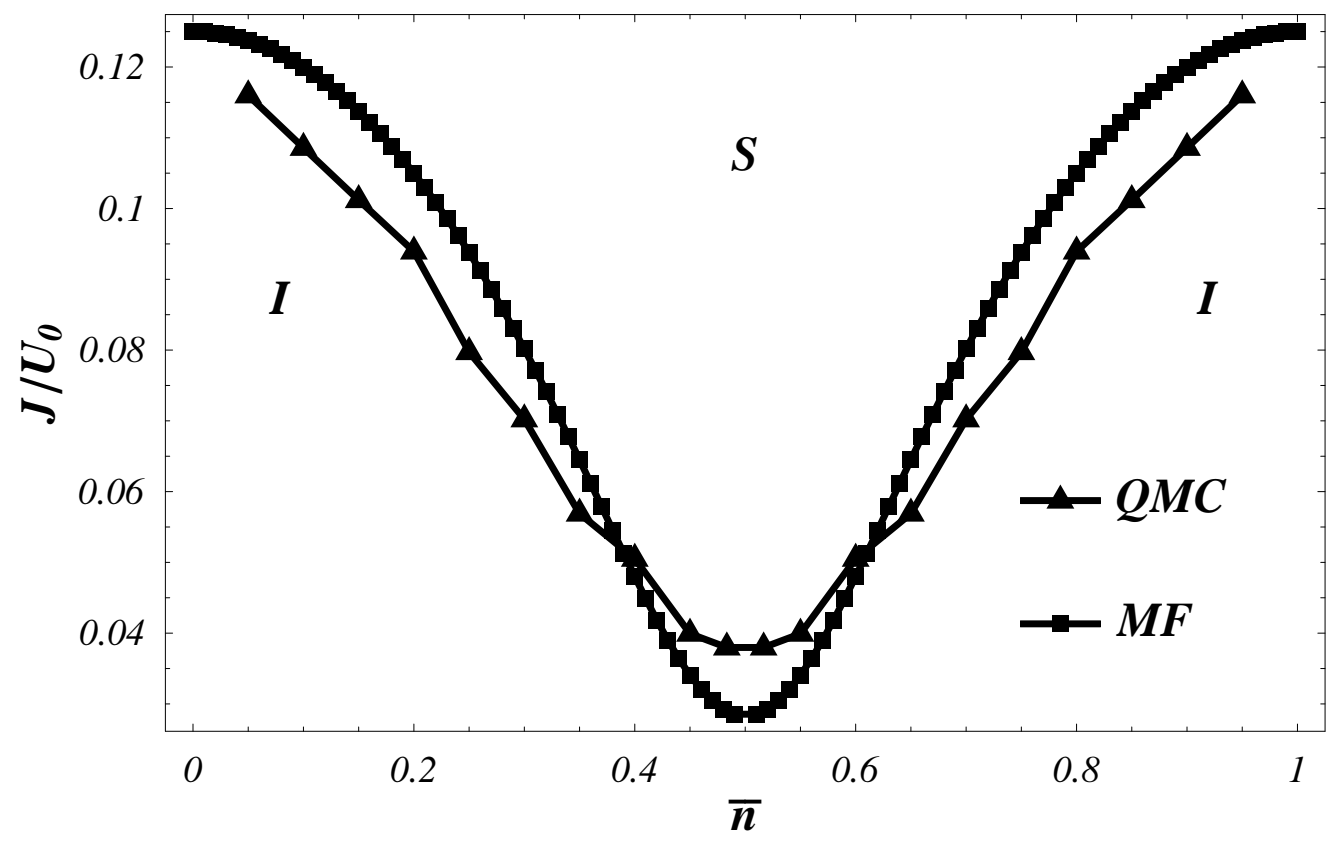

FIG. 1: Phase diagram for a model Josephson junction array described by the Hamiltonian (1), plotted as a function of the offset charge $\bar{n}$ as calculated using QMC and MF theory, in the absence of disorder. The curves show the critical value of $J / U_{0}$ separating the superconducting (S) phase from the insulating (I) phase. The temperature is $k_{B} T=0.03 U$ and the QMC calculations are done for a lattice of size $20^{3}$. The line segments simply connect the calculated points

\section{RESULTS}

We have carried out our simulations for three different system sizes: $8^{3}, 10^{3}$, and $14^{3}$, and have usually averaged over $\sim 100-500$ realizations of the disorder. For system with no disorder we studied system with a size of $20^{3}$. For each disorder realization, the system is equilibrated by slowly annealing in temperature, over about $2000 \times L^{2} L_{\tau}$ passes through the entire system for each value of $E_{J}$ considered, followed by approximately twice as many steps over which the grand canonical averages are computed.

Figure 1 shows the phase diagram for a JJA at $k_{B} T=0.3 U$ with diagonal charging energy and no disorder, as calculated using QMC as described above. For reference, we also show the same phase diagram as calculated using $\mathrm{MFT}^{6.7}$. The regions of the phase diagram denoted $S$ or $I$ correspond to the superconducting and Mott insulating regions, respectively. The two curves show the phase boundaries as calculated using MFT (squares) and QMC 
(a)
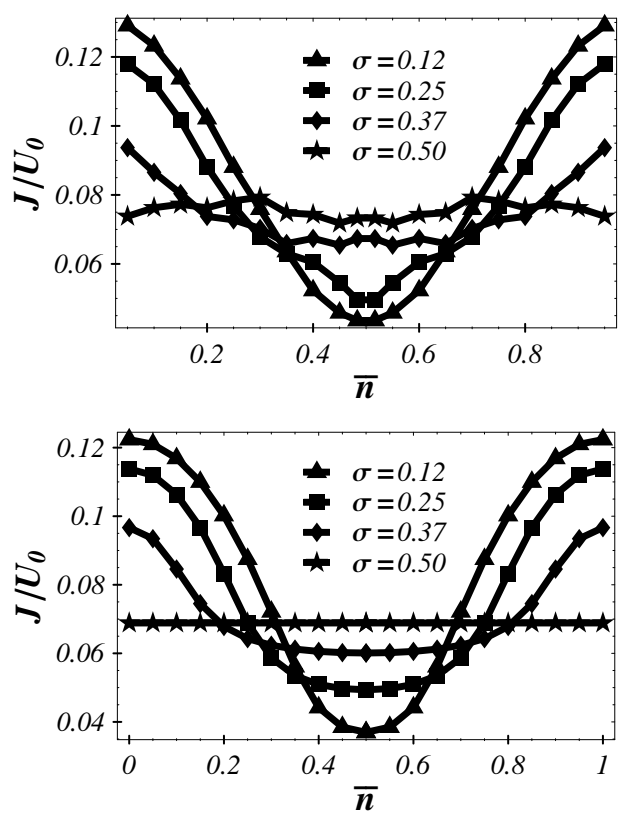

(b)
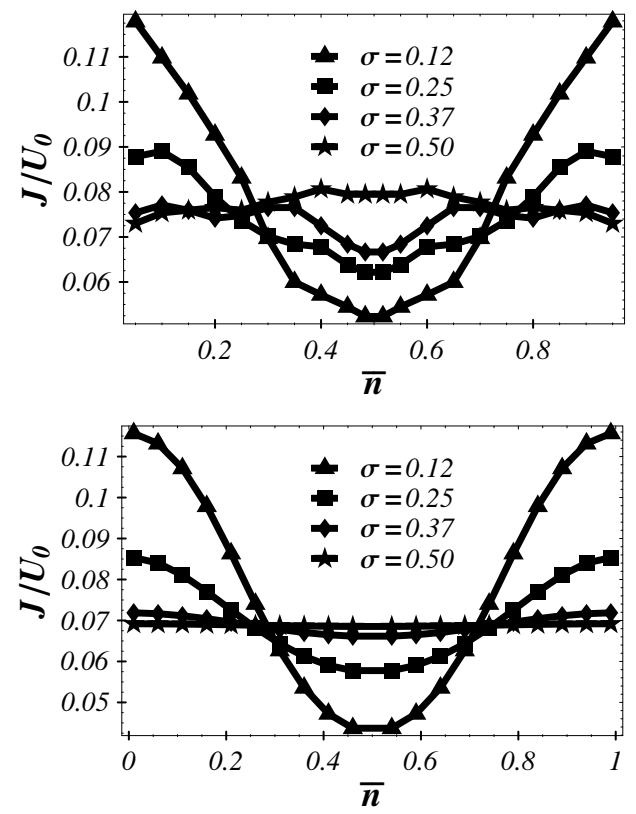

FIG. 2: (a) Phase diagram for the model Hamiltonian (1) as calculated using QMC (upper panel) and MFT theory (lower panel), including the effects of disorder. The offset charge is chosen from a uniform distribution between $\bar{n}-\sigma$ and $\bar{n}+\sigma$. For the QMC calculation, we use a $14^{3}$ lattice and fix the temperature at $k_{B} T=0.03 U_{0}$. (b) Same as (a) except that the offset charge is chosen from a Gaussian distribution of standard deviation $\sigma$ centered at $\bar{n}$. In these calculations, line segments connect calculated points.

(triangles). Although in this and later Figures we show results for $0 \leq \bar{n} \leq 1$, calculations were actually carried out only in the region $0 \leq \bar{n} \leq 0.5$, since the phase diagram must be symmetric about $\bar{n}=0.5$. Our findings here are similar to what is found by Otterlo et al in Ref. ${ }^{12}$.

We have considered two types of disorder: randomness in the offset charges $\bar{n}_{i}$, and randomness in the diagonal charging energies $U_{i i}$. Disorder in $\bar{n}_{i}$ is the analog of chemical potential disorder in the BHM, while disorder in the $U_{i i}$ 's is analogous to randomness in the mean-field on-site Coulomb energy in the BHM.

We begin by describing our results for offset charge randomness. We have carried out calculations with two different random distributions of $\bar{n}$ 's. In the first case, we choose the offset charge at each lattice site so that $\bar{n}_{i}=\bar{n}+\sigma_{i}$, where $\sigma_{i}$ is a random number uniformly 

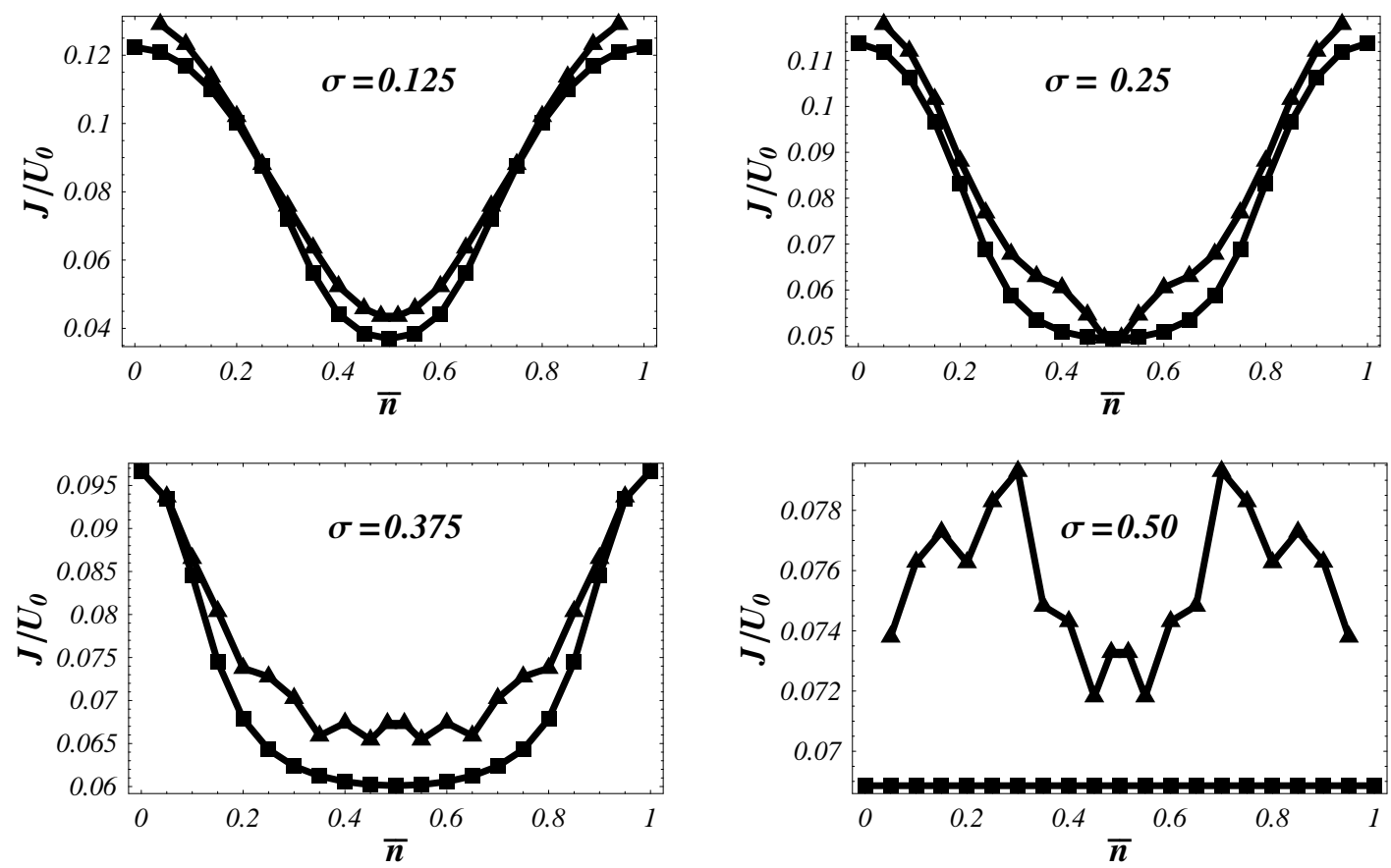

FIG. 3: Comparison of the phase diagram as calculated using QMC (triangles) and MF theory (squares), assuming a uniform distribution of $\bar{n}$ and several values of the disorder strength parameter $\sigma$, as indicated in each plot. Other parameters are the same as in Fig. 2.

distributed in the range $[-\sigma, \sigma]$, where $\sigma$ represents the strength of disorder. In the second case, we choose $\bar{n}_{i}$ to have a Gaussian distribution with a mean value of $\bar{n}$ and standard deviation $\sigma$, i.e., with a probability distribution proportional to $e^{-\left(n_{i}-\bar{n}\right)^{2} / 2 \sigma^{2}}$. In both cases, the values of $\bar{n}_{i}$ on different sites are taken as uncorrelated.

In Fig. 2 (a), we show the phase diagram for a disordered JJA, assuming a flat distribution of $\bar{n}_{i}$, and as calculated using QMC (upper panel), and MFT (lower panel). In each case, the different curves correspond to the phase diagrams for different disorder strengths, as indicated in each Figure. In Fig. 2 (b), we show the corresponding results, but for Gaussian disorder with various standard deviations $\sigma$, as indicated in the figure.

Several features are noticeable from the QMC calculations. First, at values of $\bar{n}$ close to 0 or 1 , disorder reduces the stability region of the insulating phase, whereas near $\bar{n}=1 / 2$, disorder increases the region in which the insulating phase is stable. These effects are readily understood: a finite disorder means that one is effectively calculating the phase diagram over some average region of $\bar{n}$, and therefore the sharp lobes seen in the phase 

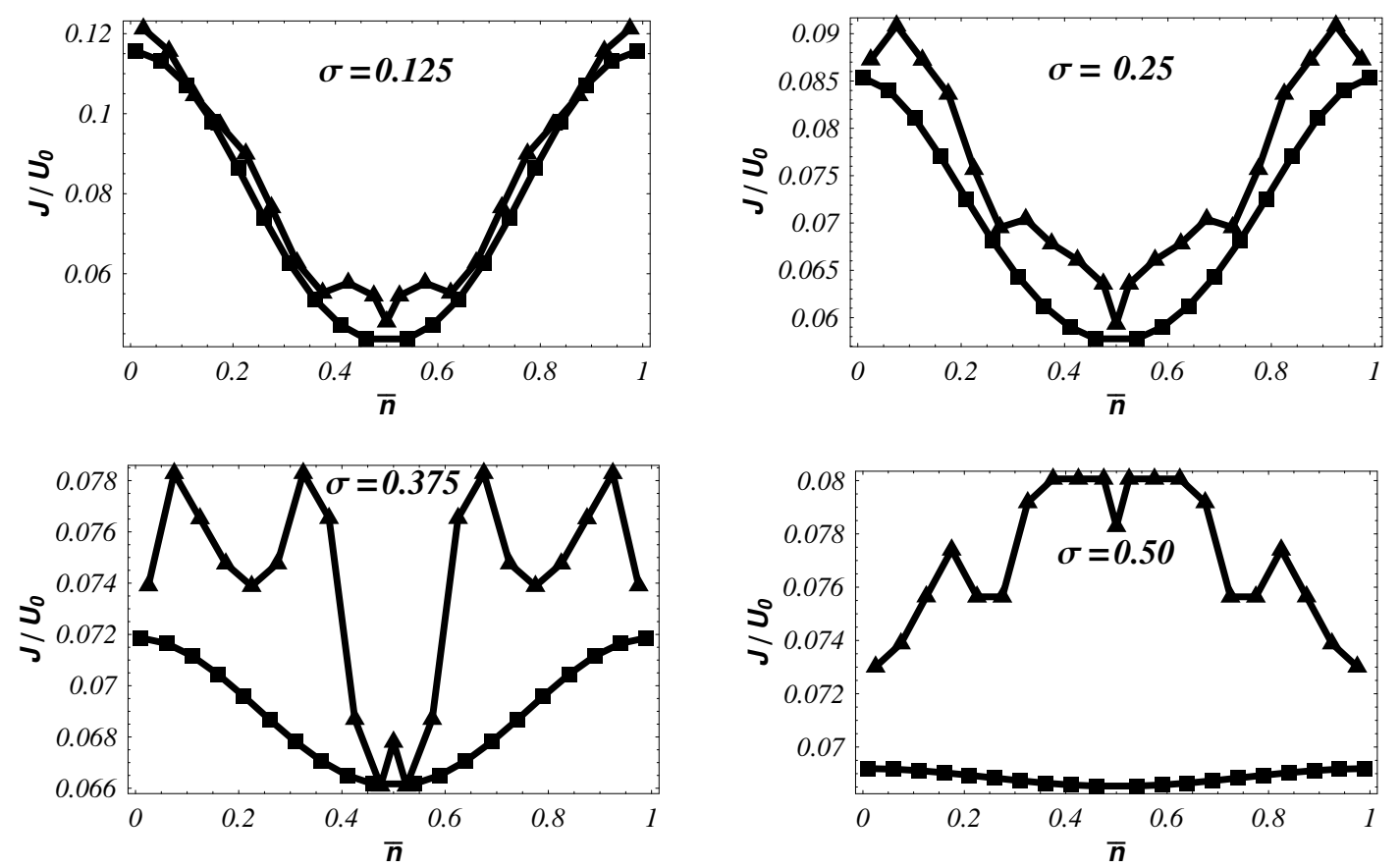

FIG. 4: Same as Fig. 3 except that the offset charge $\bar{n}$ is chosen from a Gaussian distribution of standard deviation $\sigma$.

diagram for non-random $\bar{n}$ should become less distinct as the disorder increases. Indeed, the lobe structure shrinks and tends to disappear for large values of disorder. A similar effect was also emphasized by Fisher et al for the Bose Hubbard model ${ }^{\underline{8}}$. This behavior is not surprising, because in the limit of large disorder strength $\sigma$, the Hamiltonian becomes independent of $\bar{n}$ : all values of $\bar{n}$ are therefore equivalent. (This equivalence is exact for the uniform distribution at $\sigma=0.5$.)

Results from the QMC and MFT calculations are compared in a different way in Figs. 3 and 4 for the two kinds of disorder in $\bar{n}_{i}$. As is evident from both Figures, the MFT results agree very well with those from QMC results for both types of disorder, except possibly near the tips of the lobes. Near $\bar{n}=0.5$, the critical value of $J / U_{0}$ is slightly underestimated by MFT. A similar underestimate occurs for large values of the disorder strength $\sigma$. In this case, MFT underestimates the critical value of $J / U_{0}$ by about $10 \%$.

Figure [5 shows the results of calculations in which disorder is included in the diagonal charging energy but not in the $\bar{n}_{i}$ 's. Specifically, we choose $U_{i i}$ at random from values uniformly distributed in the interval $\left[U_{0}(1-\Delta), U_{0}(1+\Delta)\right]$. We have considered several values of the disorder strength parameter $\Delta$, as indicated in the legend. Clearly, randomness 


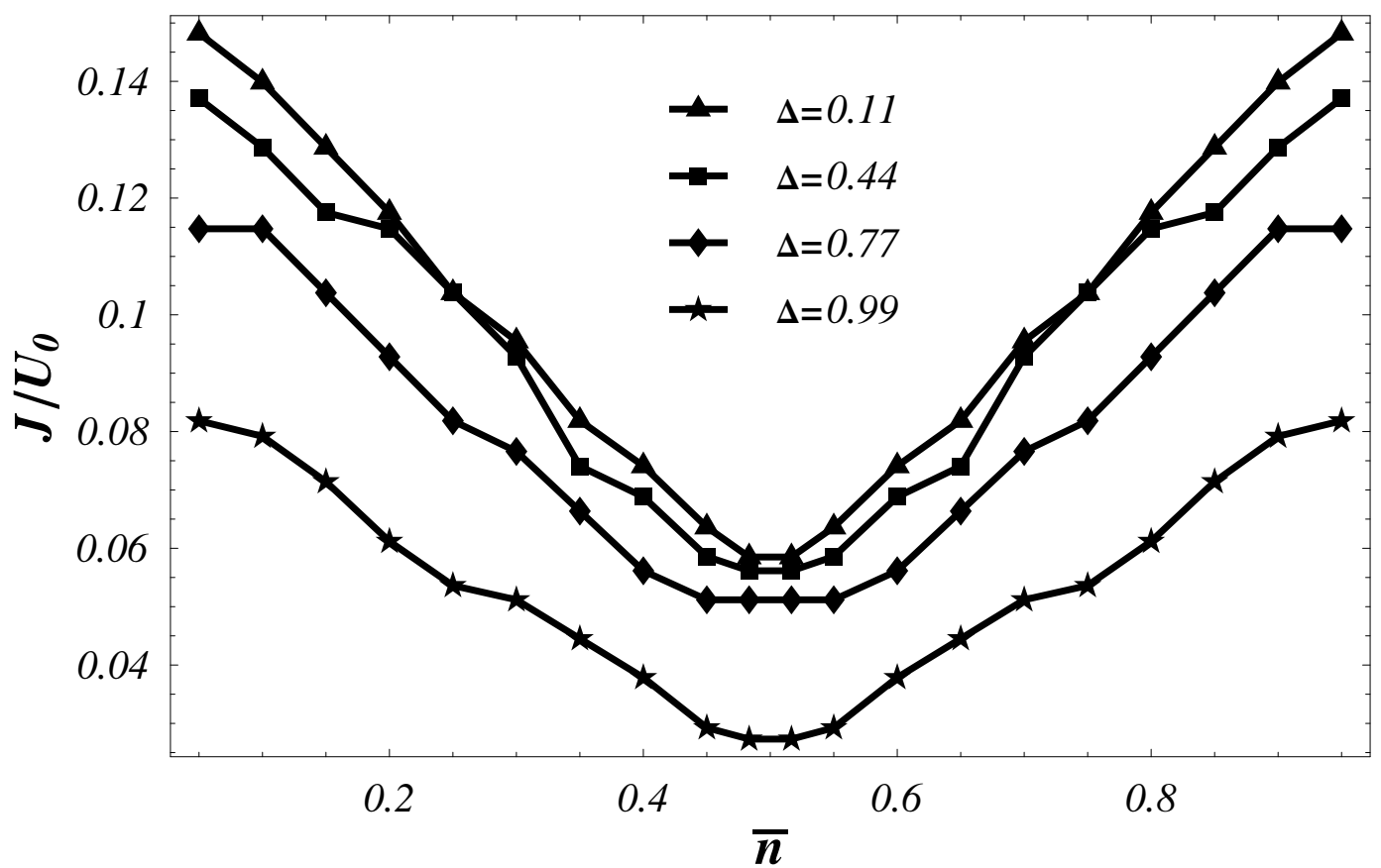

FIG. 5: Phase diagram for a disordered 2D Josephson junction array described by the Hamiltonian (1), with charging energy disorder. Only the diagonal elements $U_{i i}$ of the charging energy are nonzero; the $U_{i i}$ 's are distributed randomly and uniformly in the interval $\left[U_{0}(1-\Delta), U_{0}(1+\Delta)\right]$. The values of the disorder parameter $\Delta$ are shown in the Figure. Other parameters are the same as in Fig. 2 ,

in the $U_{i i}$ always increases the region of the phase diagram in which the $S$ phase is stable, whatever the value of $\bar{n}$. This trend is not surprising, because fluctuations in the $U_{i i}$ 's lead to greater fluctuations in the number of Cooper pairs on each island. Thus, by the uncertainty principle, the phase fluctuations should be relatively reduced, and therefore the $S$ (phase-ordered) state should become more favorable, as seen in our calculations.

\section{DISCUSSION AND SUMMARY}

In this paper, we have studied the phase diagram of a model Josephson junction array at low temperatures using quantum Monte Carlo techniques. Our goal was to analyze the effects of disorder in both the offset charges $\bar{n}_{i}$ and grain sizes (which affect the diagonal elements of the charging energy matrix, $U_{i i}$ ). For disorder in the $\bar{n}_{i}$ 's, we find that disorder favors the $S$ phase when the average offset charge $\bar{n}$ is close to an integer number of Cooper 
pairs, but that it favors the $I$ phase near $\bar{n}=1 / 2$. We also find that for large disorder, the average value $\bar{n}$ has little effect on the phase diagram at all. As for the second kind

of disorder, we find that disorder in the $U_{i i}$ 's tends to favor the $S$ phase for all values of $\bar{n}$, as shown in Fig. 5. Finally, we have found that the critical value of $E_{J} / U$ at the phase boundary, as calculated from the MFT of Grignani et al $\underline{\underline{6}}$, agrees well with that calculated using QMC (almost perfectly for small disorder and within 10\% even for large disorder). Thus, the MFT gives good results for most of the phase diagram.

\section{ACKNOWLEDGMENTS.}

This work has been supported by NSF Grant DMR01-04987. The calculations were carried out on the COE machines of the Ohio Supercomputer, with the help of a grant of time.

* Electronic address: Al-Saidi.1@osu.edu

$\dagger$ Electronic address: Stroud@mps.ohio-state.edu

1 S. L. Sondhi, S. M. Girvin, J. P. Carini, and D. Shahar, Rev. Mod. Phys. 69, 315 (1997).

2 R. S. Newrock, C. J. Lobb, U. Geigenmüller, and M. Octavio, Solid State Physics 54, 263 (2000).

3 For a recent review see, for example, R. Fazio, and H. van der Zant, Phys. Rep. 355, 235 (2001).

4 For a recent review see, Y. Makhlin, G. Schön, and A. Shnirman, Rev. Mod. Phys. 73, 357 (2001) and references therein.

5 P. W. Anderson, in "Lectures on the Many body problem" (edited by E. R. Caianello), Vol. 2, 113, (Academic 1964); B. Abeles, Appl. Sol. State Science 6, 1 (1975).

6 G. Grignani, A. Mattoni, P. Sodano, and A. Trombettoni, Phys. Rev. B 61, 11676 (2000).

7 F. P. Mancini, P. Sodano, and A. Trombettoni, Phys. Rev. B 67, 014518 (2003).

8 M. P. A. Fisher, P. B. Weichman, G. Grinstein, and D. S. Fisher, Phys. Rev. B 40, 546 (1989).

9 R. T. Scalettar, G. G. Batrouni, and G. Zimanyi, Phys. Rev. Lett. 66, 3144 (1991); G. G. Batrouni, B. Larson, R. T. Scalettar, J. Tobochnik, and J. Wang Phys. Rev. B. 48, 9628 (1993);

10 J. -W. Lee, M. -C. Cha, and D. Kim, Phys. Rev. Lett. 87, 247006 (2001); ibid. 88, 049901 (2002). 
11 Mats Wallin, Erik S. Sørensen, S. M. Girvin, and A. P. Young, Phys. Rev. B 49, 12115 (1994).

12 Anne van Otterlo and Karl-Heinz Wagenblast, Phys. Rev. Lett. 72, 3598 (1994).

13 Eric Roddick and David Stroud, Phys. Rev. B 51, 8672 (1995).

14 J. Villain, J. Phys. (Paris) 36, 581 (1975). 